\title{
Image Mining: Review and New Challenges
}

\author{
Barbora Zahradnikova, Sona Duchovicova and Peter Schreiber \\ Institute of Applied Informatics, Automation and Mechatronics, \\ Faculty of Materials Science and Technology, \\ Slovak University of Technology, \\ Hajdoczyho 1, Trnava, Slovakia
}

\begin{abstract}
Besides new technology, a huge volume of data in various form has been available for people. Image data represents a keystone of many research areas including medicine, forensic criminology, robotics and industrial automation, meteorology and geography as well as education. Therefore, obtaining specific information from image databases has become of great importance. Images as a special category of data differ from text data as in terms of their nature so in terms of storing and retrieving. Image Mining as a research field is an interdisciplinary area combining methodologies and knowledge of many branches including data mining, computer vision, image processing, image retrieval, statistics, recognition, machine learning, artificial intelligence etc. This review focuses researching the current image mining approaches and techniques aiming at widening the possibilities of facial image analysis. This paper aims at reviewing the current state of the $\mathrm{IM}$ as well as at describing challenges and identifying directions of the future research in the field.
\end{abstract}

Keywords-image mining; image classification; indexing; image retrieval;

\section{INTRODUCTION}

Due to the enormous research and development of the recent years, the lack of information has not been an issue in the most fields of human activity. On the contrary, besides new technology, there is a huge volume of data available for people. Therefore, sorting the data and obtaining specific information from databases has become of great significance. In the last decade, data mining as a research field has expanded and progress in data processing is getting both more accurate and convenient. Besides text data mining; novel data mining algorithm; web mining and social network analysis, image mining belongs to the spheres of interest.

Analysing image data forms a keystone of many research areas including medicine (evaluating MRI, interpreting XRays/CT scans), forensic criminology (fingerprint identification, face recognition), robotics and industrial automation (robotic vision), meteorology and geography (satellite imagery) as well as education (computer-aided visualization) and many other fields.

Searching information within images represents a special entity of data processing. Images as a unique category of data differ from text data in several aspects as in terms of their nature so in terms of storing and retrieving. Images have visual character, they can be represented in numerical form, however large amount of numbers is to be evaluated in order to search image databases. Finding, extracting and classifying objects from images are the basic requirements of processing an image successfully. Tools of data mining have been utilised for these tasks to be performed with increased efficiency. Nevertheless, applying data mining solely would not bring satisfactory results for image processing.

Image mining deals with extraction of implicit knowledge, image data relationship or other patterns not explicitly stored in image [1]. Unlike other image processing techniques, IM does not aim at detecting a specific pattern in images. It focuses rather on identifying and finding image patterns and deriving the knowledge from images within an image set based on the low-level (pixel) information. As a research field, it has developed to an interdisciplinary area combining knowledge and tools of data mining, databases, computer vision, image processing, image retrieval, statistics, recognition, machine learning, artificial intelligence, etc. Image mining process consists of several components including

- image analysis covering image preprocessing, object recognition and feature extraction,

- image classification,

- image indexing,

- image retrieval,

- data management.

A number of approaches for each of the above mentioned procedures have been proposed. Yet, image processing stays a domain where humans still can outperform computer.

This paper aims at reviewing the steps of image mining, the most often utilised techniques for the individual subprocesses of IM and at identifying the major current issues and challenges in image mining.

\section{IMAGE ANALYSIS}

Image analysis is an inevitable step of image Mining. The analysis is often said to be a pre-processing stage of the image minig [2]. The objective of analysing an image is to find and extract all relevant features required to represent an image.

\section{A. Image Preprocessing}

Image preprocessing is an initial step of processing images. It is utilised for improving the quality of an image before object detection algorithms are applied. Normalising images is usually performed in order to reduce noise and/or enhance resolution of an image. Different pre-processing procedures 


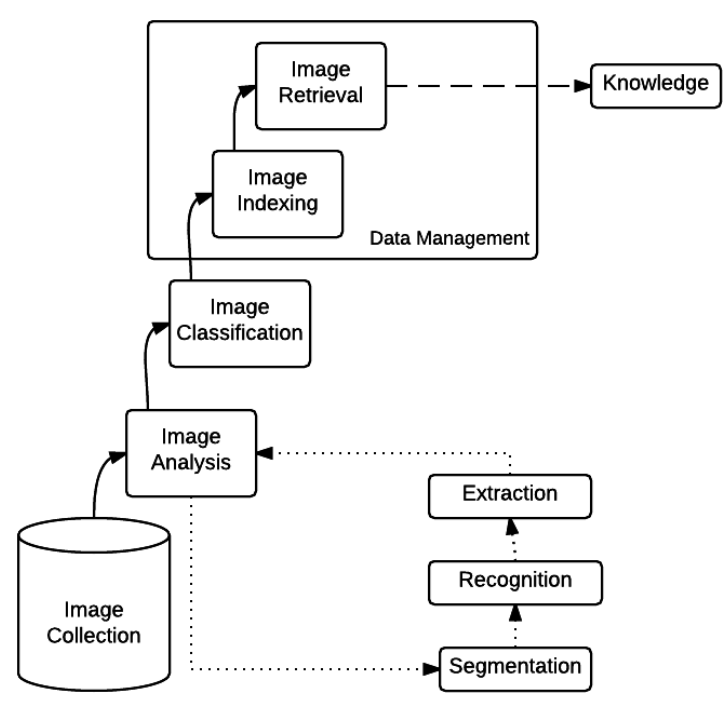

Fig. 1: Traditional Image Mining Procedure

might by employed including average, median and wiener filtering for lowering the impact of noise and interpolation based Discrete Wavelet Transform (DWT) and Multiresolution Image Fusion to enhance the resolution [3], [4], [5].

\section{B. Object Recognition}

Object recognition is a step resulting in segmentation of an image. It focuses on identifying objects in an image and dividing an image into several regions accordingly. It is a task which until recently was considered the main objective of image processing. Visual objects are to be detected from an image according to a model. The model represents certain patterns obtained as an outcome of applying a training algorithm on the training sample. For this purposes, supervised machine learning needs to be deployed.

Once the objects can be identified within an image, it can be segmented into subareas. Berlage distinguishes three segmentation approaches [6]:

1) Marker-based segmentation - Objects are represented by an area covered by a marker. The object to be detected is identified based on labelling the space within an image.

2) Object-based segmentation - Objects are identified without the boundaries being exactly determined.

3) Contour-based segmentation - The contours need to be matched pixel precisely.

Many algorithms for object identification have already been proposed and are exploited in practice. Face and smile detection algorithms utilised by cameras or recognition systems, tool detection applied for improving robotic vision, tumour detection from MRI are just examples of successful deploying the recognition/detection systems. Still, there are unsolved issues in object detection. Additionally, currently, the task is not only to detect an object, but also to extract, mark or in other way represent the pixel information for further processing.

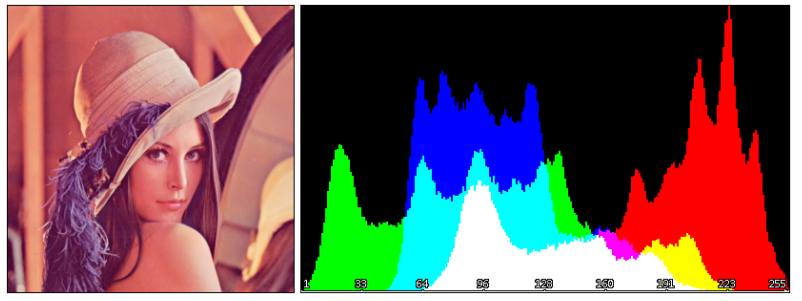

Fig. 2: Example of Colour Histogram [10]

\section{Feature Extraction}

Extracting features stands for a process of compressing the information derived from identified objects into a set of attributes. Both local and global descriptors may be used for representing the image. Global descriptors are easier to compute and do not tend to segmentation errors. In comparison, local descriptors provide much precise representation and might discover even subtle patterns [7]. Features are usually represented numerically and provide complex mathematical representation of an image. They describe objects in terms of shape, texture and/or colour, etc.

As a result of attempt to unify the way video and audio are described, MPEG-7 was standardised as ISO/IEC 15938 (International Organization for Standardization / International Electro-technical Commission). This standard is often referred to as Multimedia content description interface. According to Martinez, visual descriptors are divided into the following description tools [7]:

1) Basic Elements - Grid layout, time series, 2D3D multiple view, spatial $2 D$ coordinates, and temporal interpolation are examples of tools further used by other descriptors.

2) Colour - Colour histogram is the most commonly used description. It enables easy computing and provides effective characteristics of colour distribution in an image. Furthermore, as a descriptor, colour histogram is invariant to rotation and translation [8]. Colour moments can be also used as descriptors. They are usually applied as the first filter before applying other, more sophisticated methods for image retrieval [9]. Colour Space, Colour Quantization, Scalable Colour, Dominant Colour, Colour Layout, Colour Structure, and Group-of-Frames/Groupof-Pictures Colour are described as colour descriptors by [7].

3) Texture - Texture is a visual description tool characterising the visual patterns occurring in images based on the granularity, directionality and repetitiveness. Statistical methods used for defining image intensity include e.g. Tamura Features, Shift Invariant Principal Component Analysis, Fourier Power Spectra and Markov Random Field as well as multi-resolution filtering techniques Gabor and Wavelet transform [8]. The descriptors of texture defined by MPEG-7 are Homogeneous Texture, Non-Homogeneous Texture (Edge histogram), and Texture Browsing [7].

4) Shape - Boundary-based (rectilinear shapes, polygonal approximation, finite element models, Fourrier-based), region-based (statistical moments) and $3 D$ Shape methods are the most often utilised techniques for shape descrip- 


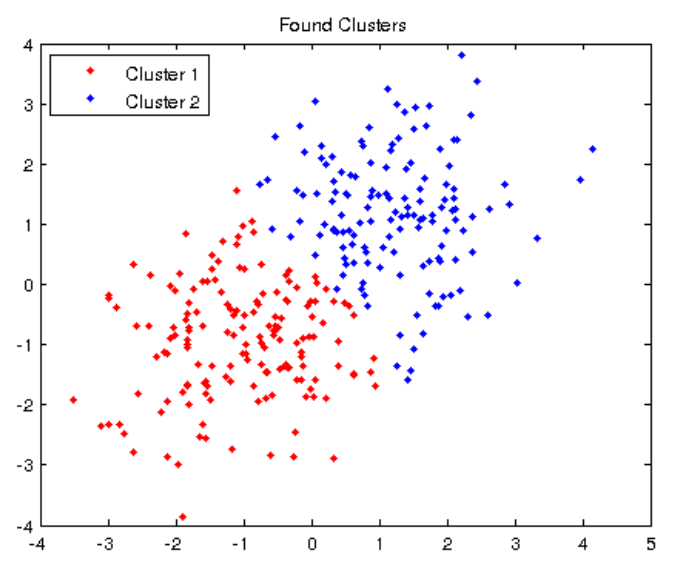

Fig. 3: Example of Data Clustering

tion [8], [7].

5) Motion (for video) - Motion Activity, Camera Motion, Parametric Motion, and Motion Trajectory

6) Location - Region Locator and Spatio-Temporal Locator.

\section{IMAGE CLASSIFICATION}

The objective of classification is to categorise objects detected in an image. Currently, classification objects is an extensively researched domain. Different approaches have been proposed and tested. However, the field remains in its infancy and categorising into non-pre-defined classes is still an issue to be solved. The researched methods of categorising objects as described by [6], [8], [11] are:

\section{A. Supervised Classification}

Supervised classification is the original approach of categorising images. The objective is to divide the detected objects into pre-defined categories. Methods of machine learning (decision tree, rule-based classification, support vector machines,neural networks) are applied on training the system based on the the labelled (pre-classified) samples and followingly, on labelling new images using the obtained (trained) classifiers.

\section{B. Image Clustering}

In contrast to standard classification methods, clustering represents unsupervised categorization of objects. The objects are grouped into clusters based on the similarity, not on the basis of predefined labels. Cluster analysis aims at searching for common characteristics without knowing the exact data types. It is oriented on decomposing images into groups of objects similar to each other and different from the other objects as much as possible. The similarity is evaluated based on the calculated features (texture, shape, colour,...).

Hierarchical clustering, partition based clustering, mixture resolving, nearest neighbour clustering, fuzzy clustering, evolutionary clustering are some of approaches used for unsupervised categorization. After accomplishing the clustering process (dividing the objects into clusters), an expert form the particular field is needed to identify the individual categories (clusters).

\section{Data Management}

Images cover a huge amount of information. Depending on the way of storing and indexing images, various knowledge might be searched and retrieved from an image database.

\section{A. Storing Images}

Zhang et al. identified several differences between image databases and relational databases pointing put the misusing and misunderstanding the term of Image Mining [11]. IM cannot be understood barely as applying data mining techniques on images, as compared to relational databases, there are important differences in handling images:

- Relativity of values - Images can be numerically represented, however, in contrast to relational databases, the values are only significant in a certain context.

- Dependency on the spatial information - When working with image databases, the position of individual pixels is an inevitable factor for correct interpretation of image content.

- Multiple interpretations - In comparison with relational databases, image databases are more difficult to handle, as the same patterns derived from images might have multiple interpretations depending on the context and position.

There are different ways of storing images. Several compression formats (JPEG, MPEG 7, DICOM) store the meta data in one file with an image. According to [6]), this approach might result in difficulties with analysing images. Databases of such images are not the most suitable candidates for data mining, as they are not optimised for time- and memoryconsumption when performing image retrieval.

[12] and [13] propose separating the metadata into relational databases from raw images stored in file system in order to provide faster and more efficient image management. This technique needs additional referencing, which requires a certain degree of self-discipline.

Complex data management in the form of object-oriented databases is also a solution proposed by [14]. However, standardising is required to enable broader exploitation of the method.

\section{B. Image Indexing and Image Retrieval}

In order to enable retrieving images from databases efficiently, a suitable indexing is required. Relational databases provide indexing based on primary and secondary keys. This approach is not applicable when mining image databases, as the image retrieval is most often similarity-based. $K-D-B$ tree, $R$-tree, $R^{*}$-tree, $R+$ tree, $S R$-tree, $T V$-tree, $X$-tree and iMiniMax are the most utilised indexing methods as described by ([11], [15], [16], [17], [18], [19], [20])

The retrieval techniques as described by [21] cover: 


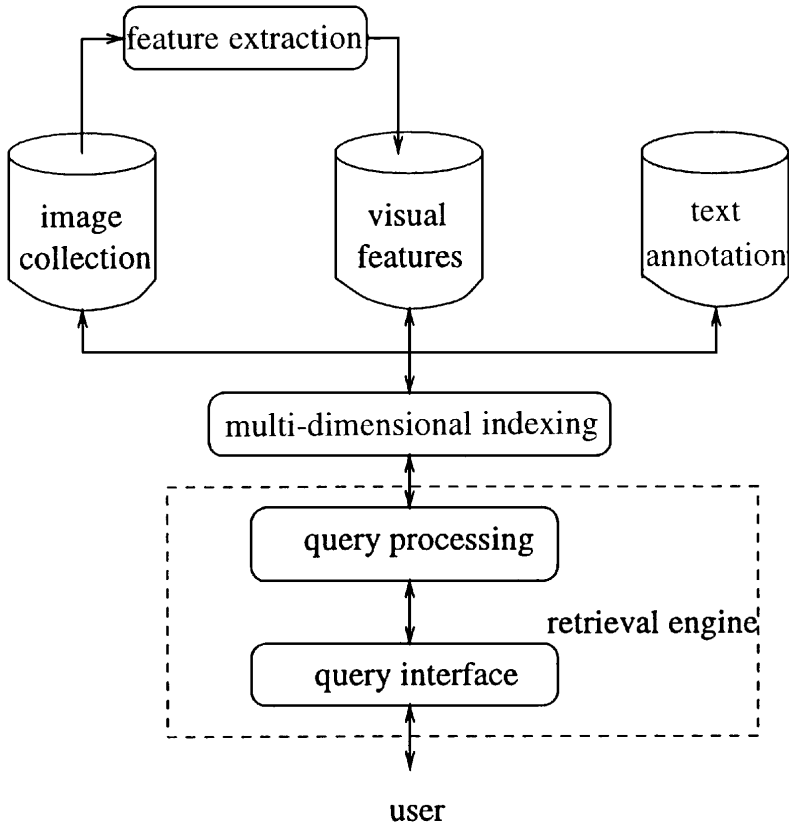

Fig. 4: Example of Multimodal Retrieval System for Image Mining [16]

- Query by Associate Attributes - Retrieving images based on the attributes stored as metadata

- Query by Description - Description of the context stands for key words assigned to images (e.g. in file names)

- Query by Content - Organising pictures according to their visual content (according to the detected features, such as texture, shape, colour; according to the similarity, etc. )

Many later applications are focused on combining the above mentioned methods in order to enable more specific and comfortable search for particular data.

Multimodal retrieval was proposed for managing several kinds of unstructured data including image, video, audio and text simultaneously by [22]. The proposed algorithm enables both retrieving data based on visual features and text models. Framework deploying deep learning architecture was proposed by [23] as a tool for improving accuracy of image retrieval in medical image data management. [24] also developed a text based indexing system for mammographic image retrieval and classification. Extracting an accurate information from large amount of data is accomplished leaning on Bayesian Naive classifier.

\section{Challenges}

Automated Image Analysis and consequent Knowledge Acquisition based on computer-driven processing images have tremendous potential. The research is still at the beginning and many areas need further investigation. There are many issues to be solved in order for the computer to be able to efficiently analyse images and to derive knowledge accordingly:
- Moving away from low-level pixel representation of images is evident. For successful image mining, developing representation of images able to encode the contextual information hidden in an image is crucial.

- A necessary step in Image Mining is classification of the obtained patterns. Automatic deriving of appropriate decision criteria for clustering represents an obstacle still difficult to be overcome.

- Proposing a suitable indexing method is also of concern. There is need for standardising the procedures of indexing and retrieving knowledge from images.

- A query language able to request both visual patterns and textual information (metadata related to an image) needs to be developed and unified.

- World Wide Web can be seen as an image database containing huge volume of images. Beyond the images, there is an unlimited amount of information. Analysing the Web and retrieving particular/searched knowledge from the images stored online currently represents the major challenge for image mining and image processing as such.

\section{CONCLUSION}

The goal of this paper was to emphasize the fact, that nowadays, users (including doctors, meteorologists, investigators, teachers and students, etc.) need to face and utilize an incredible amount of pictures stemming from the Internet or various private and commercial databases. The review aims at stressing out the need of automating their processing and classification with the purpose of obtaining particular information/knowledge from an image collection.

Following the objectives, Image Mining was described as an interdisciplinary research area, the particular steps needed for IM were reviewed and the commonly exploited IM techniques were summarised.

The tasks of introducing automated detection of unknown patterns in image sets and deriving contextual information based on these patterns were defined as the main purposes of Image Mining. Accordingly, at each IM level, the benefits and bottlenecks of individual techniques pointing out the future focusing were identified. Besides, the final part of the paper outlines the challenges to be faced within the future research.

\section{ACKNOWLEDGMENT}

This publication is the result of implementation of the project VEGA 1/0673/15: Knowledge discovery for hierarchical control of technological and production processes supported by the VEGA.

\section{REFERENCES}

[1] M. C. Burl, C. Fowlkes, and J. Roden, "Mining for image content," Systemics, cybernetics, and informatics/information systems: analysis and synthesis, 1999.

[2] A. Hema and E. Annasaro, "A survey in need of image mining techniques," International Journal of Advanced Research in Computer and Communication Engineering (IJARCCE), 2013. 
[3] S. Rajeshwari and T. S. Sharmila, "Efficient quality analysis of mri image using preprocessing techniques," in Information \& Communication Technologies (ICT), 2013 IEEE Conference on. IEEE, 2013, pp. 391-396.

[4] V. Starovoitov, D. Samal, and D. Briliuk, "Image enhancement for face recognition," in International Conference on Iconics, 2003.

[5] M. Stanković, B. J. Falkowski, D. Janković, and R. S. Stanković, "Calculation of the paired haar transform through shared binary decision diagrams," Computers \& Electrical Engineering, vol. 29, no. 1, pp. 1324,2003

[6] T. Berlage, "Analyzing and mining image databases," Drug discovery today, vol. 10, no. 11, pp. 795-802, 2005.

[7] J. M. Martínez, R. Koenen, and F. Pereira, "Mpeg-7: the generic multimedia content description standard, part 1," MultiMedia, IEEE, vol. 9, no. 2, pp. 78-87, 2002.

[8] N. Mishra and D. S. Silakari, "Image mining in the context of content based image retrieval: a perspective," IJCSI International Journal of Computer Science Issues, vol. 9, no. 4, pp. 98-107, 2012.

[9] R. da Silva Torres and A. X. Falcão, "Content-based image retrieval Theory and applications." RITA, vol. 13, no. 2, pp. 161-185, 2006.

[10] "Understanding how the image colors are distributed," http://opensource.graphics/, accessed: 2010-05-20.

[11] J. Zhang, W. Hsu, and M. L. Lee, "Image mining: Issues, frameworks and techniques," in Proceedings of the 2nd ACM SIGKDD International Workshop on Multimedia Data Mining (MDM/KDD'01). University of Alberta, 2001.

[12] M. E. Martone, S. Zhang, A. Gupta, X. Qian, H. He, D. L. Price, M. Wong, S. Santini, and M. H. Ellisman, "The cell-centered database," Neuroinformatics, vol. 1, no. 4, pp. 379-395, 2003.

[13] S. Manley, N. R. Mucci, A. M. De Marzo, and M. A. Rubin, "Relational database structure to manage high-density tissue microarray data and images for pathology studies focusing on clinical outcome: the prostate specialized program of research excellence model," The American journal of pathology, vol. 159, no. 3, pp. 837-843, 2001.

[14] B. Diallo, F. Dolidon, J.-M. Travere, and B. Mazoyer, "B-spid: An object-relational database architecture to store, retrieve, and manipulate neuroimaging data," Human brain mapping, vol. 7, no. 2, pp. 136-150, 1999.

[15] D. Robinson and L. R. Foulds, "Comparison of phylogenetic trees," Mathematical Biosciences, vol. 53, no. 1, pp. 131-147, 1981.

[16] Y. Rui, T. S. Huang, and S.-F. Chang, "Image retrieval: Current techniques, promising directions, and open issues," Journal of visual communication and image representation, vol. 10, no. 1, pp. 39-62, 1999.

[17] N. Katayama and S. Satoh, "The sr-tree: An index structure for high-dimensional nearest neighbor queries," in ACM SIGMOD Record, vol. 26, no. 2. ACM, 1997, pp. 369-380.

[18] N. Beckmann, H.-P. Kriegel, R. Schneider, and B. Seeger, The $R^{*}$-tree: an efficient and robust access method for points and rectangles. ACM, 1990, vol. 19, no. 2.

[19] S. Berchtold, D. A. Keim, and H.-P. Kriegel, "The x-tree: An index structure for high-dimensional data," Readings in multimedia computing and networking, vol. 451, 2001.

[20] B. C. Ooi and K.-L. Tan, "B-trees: bearing fruits of all kinds," in Australian Computer Science Communications, vol. 24, no. 2. Australian Computer Society, Inc., 2002, pp. 13-20.

[21] R. Kazman and J. Kominek, "Information organization in multimedia resources," in Proceedings of the 11th annual international conference on Systems documentation. ACM, 1993, pp. 149-162.

[22] J. Luo, B. Lang, C. Tian, and D. Zhang, "Image retrieval in the unstructured data management system audr," in E-Science (e-Science), 2012 IEEE 8th International Conference on. IEEE, 2012, pp. 1-7.

[23] S. Liu, S. Liu, W. Cai, H. Che, S. Pujol, R. Kikinis, M. Fulham, and D. Feng, "High-level feature based pet image retrieval with deep learning architecture," Journal of Nuclear Medicine, vol. 55, no. supplement 1, pp. 2028-2028, 2014.

[24] A. Farruggia, R. Magro, and S. Vitabile, "A text based indexing system for mammographic image retrieval and classification," Future Generation Computer Systems, vol. 37, pp. 243-251, 2014. 